\title{
Interference-induced terahertz transparency in a semiconductor magneto-plasma
}

\author{
X. Wang ${ }^{1}$, A. A. Belyanin' ${ }^{2}$, S. A. Crooker ${ }^{3}$, D. M. Mittleman' ${ }^{1}$ and J. Kono ${ }^{1 \star}$
}

\begin{abstract}
Maximum modulation of light transmission occurs when an opaque medium is suddenly made transparent. This phenomenon occurs in atomic and molecular gases through different mechanisms ${ }^{1,2}$, whereas much room remains for further studies in solids ${ }^{3-5}$. A plasma is an illustrative system showing opacity for low-frequency light, and light-plasma interaction theory provides a universal framework to describe diverse phenomena including radiation in space plasmas ${ }^{6}$, diagnostics of laboratory plasmas ${ }^{7}$ and collective excitations in condensed matter ${ }^{8}$. However, induced transparency in plasmas remains relatively unexplored ${ }^{9}$. Here, we use coherent terahertz magneto-spectroscopy to reveal a thermally and magnetically induced transparency in a semiconductor plasma. A sudden appearance and disappearance of transmission through electron-doped InSb is observed over narrow temperature and magnetic field ranges, owing to coherent interference between left- and right-circularly polarized terahertz eigenmodes. Excellent agreement with theory reveals long-lived coherence of magneto-plasmons and demonstrates the importance of coherent interference in the terahertz regime.
\end{abstract}

The free electrons in the conduction band of doped narrow-gap semiconductors, for example, InSb, InAs and $\mathrm{HgCdTe}$, behave as classic solid-state plasmas and have been examined through a number of infrared spectroscopy studies ${ }^{10,11}$. Owing to the low electron densities achievable in these materials and to the electrons' small effective mass and high mobility, most of the important energy scales (the cyclotron energy $\hbar \omega_{c}$, the plasma energy $\hbar \omega_{p}$, the Fermi energy $E_{\mathrm{F}}$, intra-donor transition energies and so on) can all lie within the same narrow energy range from $\sim 1$ to $10 \mathrm{meV}$, or the terahertz frequency range ( $1 \mathrm{THz}$ is equivalent to $4.1 \mathrm{meV}$ ). The interplay between these material properties, which are tunable with magnetic field, doping density and/or temperature, make doped narrow-gap semiconductors a useful material system in which to probe and explore new phenomena that can be exploited for future terahertz technology ${ }^{12-14}$.

Here, we have used a time-domain terahertz magnetospectroscopy system ${ }^{15}$ (see the Methods section) with a linearly polarized, coherent terahertz beam to investigate magnetoplasmonic effects in a lightly $n$-doped InSb sample that shows a sharp plasma edge at $\sim 0.3 \mathrm{THz}$ at zero magnetic field as well as sharp absorption and dispersion features around the cyclotron resonance $\left(\omega_{\mathrm{c}} / 2 \pi \sim 2 \mathrm{THz} \mathrm{T}^{-1}\right)$. These spectral features can be sensitively controlled by changing the magnetic field and temperature, owing to the very small effective masses of electrons and low thermal excitation energy in this narrow-gap semiconductor. Furthermore, long decoherence times (up to $40 \mathrm{ps)}$ of electron cyclotron oscillations give rise to sharp interference fringes and coherent beating between different normal modes (coupled photon-magneto-plasmon excitations) of the semiconductor plasma, which can be revealed by polarizationsensitive measurements.

We found that the transmission of terahertz radiation through this plasma sensitively changes with the temperature, magnetic field and frequency. As an example, we show the temperature (Fig. 1a-c) and magnetic field (Fig. 1d-f) dependence of terahertz transmittance spectra. A striking feature in both Fig. 1a and Fig. 1d is a narrow range of temperature (Fig. 1a) and magnetic field (Fig. 1d) where the transmission of terahertz light is high, which we refer to as thermally induced transparency and magnetically induced transparency, respectively. Figure $1 \mathrm{~b}$ shows a full contour map of the transmittance as a function of frequency $(0.12-2.6 \mathrm{THz})$ and temperature $(2-240 \mathrm{~K})$ at a fixed magnetic field of $0.9 \mathrm{~T}$. Figure 1c shows a calculated contour plot of the transmittance, based on a model to be discussed later. The spectra shown in these figures are characterized by two sharp 'spikes' in transmission (peaked at $\sim 1$ and $\sim 2 \mathrm{THz}$ ) that are sandwiching a relatively flat transmission region (or a plateau) between them. The two spikes of transmission are stable with increasing temperature only up to $\sim 150 \mathrm{~K}$, where they suddenly shift in frequency, one moving down and the other moving up. As a result, in a temperature region of $150-200 \mathrm{~K}$ there are two bands of transmission stretching towards both ends of our spectral range (see Fig. 1b). A horizontal cut of the contour at $0.25 \mathrm{THz}$ is shown in Fig. 1a. Similarly, Fig. 1e and f show, respectively, measured and calculated contour plots of the transmittance as a function of frequency and magnetic field at a fixed temperature of $40 \mathrm{~K}$. A horizontal cut of the contour at $0.25 \mathrm{THz}$ is shown in Fig. 1d. In the following, we show how the induced transparency and opacity arise from coherent terahertz interference in a low-density magneto-plasma.

At zero magnetic field, the only spectral feature appearing in our InSb samples is the plasma edge at the plasma frequency $\omega_{\mathrm{p}} / 2 \pi=\left[\mathrm{e}^{2} n /\left(m^{*} \kappa \varepsilon_{0}\right)\right]^{1 / 2} / 2 \pi \approx 0.3 \mathrm{THz}$, where the freeelectron density $n=2.3 \times 10^{14} \mathrm{~cm}^{-3}$, the electron effective mass $m^{*}=0.014 m_{0}$ at the band edge, $m_{0}=9.11 \times 10^{-31} \mathrm{~kg}$ is the mass of free electrons in vacuum, the lattice dielectric constant $\kappa=16$ and $\varepsilon_{0}=8.85 \times 10^{-12} \mathrm{~F} \mathrm{~m}^{-1}$ is the permittivity of vacuum. When a magnetic field is applied along the wave propagation direction (that is, the Faraday geometry), the incident linearly polarized terahertz wave propagates in the sample as a superposition of the two transverse normal modes of the magneto-plasma: the left-circularly polarized mode, called the 'extraordinary' or cyclotron resonance active (CRA) wave, and the right-circularly polarized mode, called the 'ordinary' or cyclotron resonance inactive (CRI) wave (see Fig. 2a). The CRA mode couples with the cyclotron motion of electrons. With increasing magnetic field,

${ }^{1}$ Department of Electrical and Computer Engineering, Rice University, Houston, Texas 77005, USA, ${ }^{2}$ Department of Physics, Texas A\&M University, College Station, Texas 77843, USA, ${ }^{3}$ National High Magnetic Field Laboratory, Los Alamos, New Mexico 87545, USA. *e-mail: kono@rice.edu. 
a

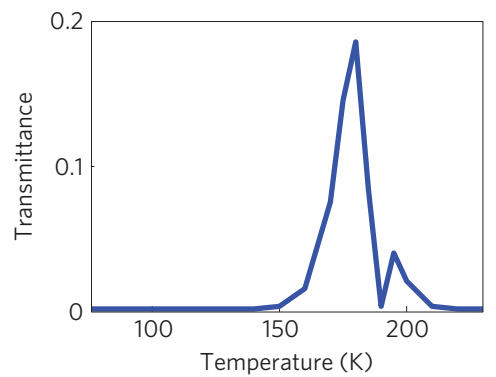

b

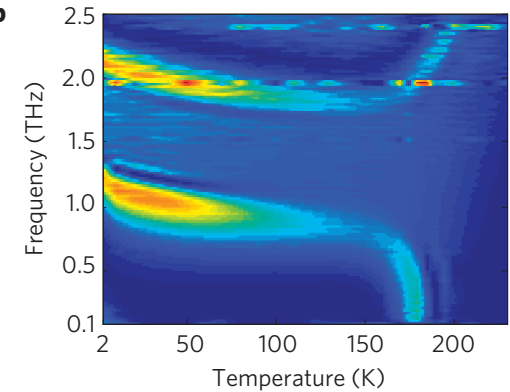

c

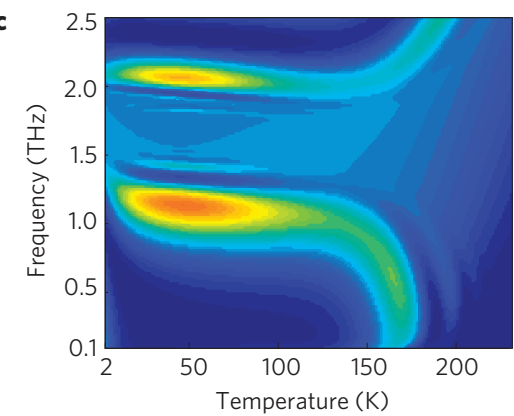

d

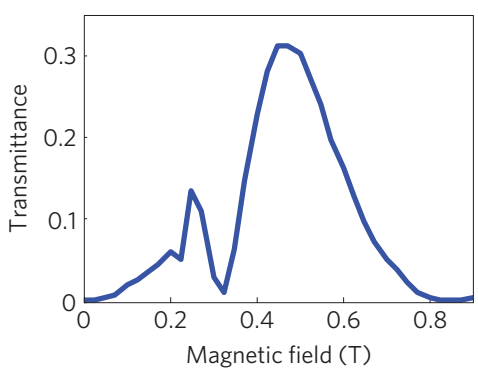

e

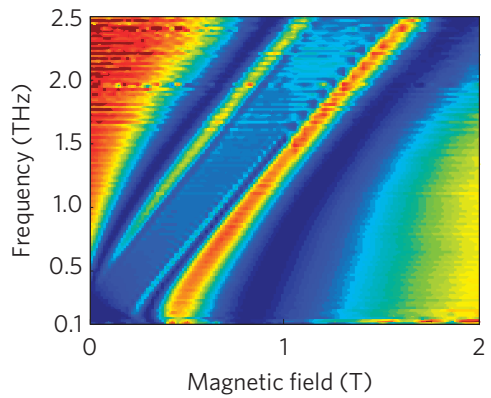

f

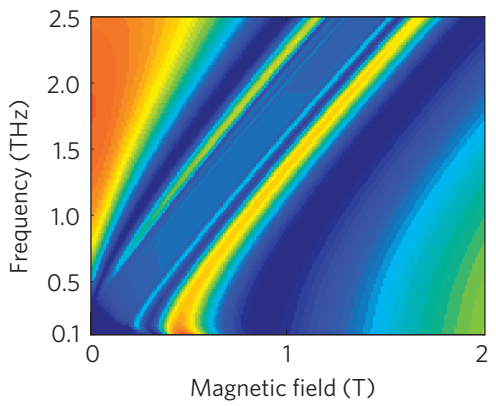

Figure 1 | Temperature dependence of terahertz transmittance spectra for lightly doped InSb in a magnetic field. a, Transmittance versus temperature at $0.25 \mathrm{THz}$ at a magnetic field of $0.9 \mathrm{~T}$ (corresponding to a horizontal cut in the contour map of $\mathbf{b}$ ), showing thermally induced transparency. b,c Measured (b) and calculated (c) transmittance contour as a function of temperature (2-240 K) and frequency $(0.12-2.6 \mathrm{THz})$ at a fixed magnetic field of $0.9 \mathrm{~T}$. d, Transmittance versus magnetic field at $0.25 \mathrm{THz}$ at a temperature of $40 \mathrm{~K}$ (corresponding to a horizontal cut in the contour map of e), showing magnetically induced transparency. e, $\mathbf{f}$ Measured (e) and calculated (f) transmittance contour as a function of magnetic field ( $0-2 \mathrm{~T})$ and frequency $(0.12-2.6 \mathrm{THz})$ at a fixed temperature of $40 \mathrm{~K}$.

the plasma edge splits into the two magneto-plasmon frequencies given by

$$
\omega_{ \pm} \cong \frac{1}{2}\left(\sqrt{\omega_{\mathrm{c}}^{2}+4 \omega_{\mathrm{p}}^{2}} \pm \omega_{\mathrm{c}}\right)
$$

for the CRA $(+)$ and CRI $(-)$ modes, respectively, as plotted in Fig. 2b. These are the characteristic frequencies at which the refractive index becomes zero (neglecting dissipation). As the magnetic field increases, the frequency $\omega_{+}$asymptotically approaches the electron cyclotron frequency $\omega_{\mathrm{c}}$ (the dotted line in Fig. 2b), whereas $\omega_{-}$monotonically decreases and approaches zero.

On the basis of these considerations, the measured terahertz magneto-transmittance spectra at low temperatures can be theoretically reproduced. We modelled the terahertz response of the InSb sample through a dielectric tensor for a classical magnetoplasma $^{10,16,17}$ for both electrons and holes, including the effect of conduction-band non-parabolicity within the Kane model ${ }^{11}$. The phonon contribution to the dielectric permittivity was taken into account within the harmonic-oscillator approximation ${ }^{18}$. The contributions of holes and phonons turned out to be small, although non-negligible. The electron scattering rate was calculated, taking into account all relevant processes over the entire range of temperatures and magnetic fields (see Supplementary Information for details of our calculations of the carrier scattering rate and mobility as a function of temperature). Figure $3 a$ and $b$ show calculated $40 \mathrm{~K}$ transmittance spectra for only CRA (that is, $\left|E_{\mathrm{te}}\right|^{2}$ only) and only CRI (that is, $\left|E_{\mathrm{to}}\right|^{2}$ only), respectively, where $E_{\mathrm{te}}$ and $E_{\mathrm{to}}$ are the transmitted electric fields of the CRA and CRI modes, respectively (see Fig. 2a). The CRA wave experiences strong absorption and dispersion in the vicinity of $\omega_{+}$. On the other hand, the transmission of the CRI mode is nearly flat and featureless everywhere except at very low frequencies. Simple addition of the two (that is, $\left|E_{\mathrm{te}}\right|^{2}+\left|E_{\mathrm{to}}\right|^{2}$ ), however, does not produce any of the experimentally observed spectral features, except the clear plasma edge at zero field, as shown in Fig. $3 c$.

The $x$ component of the transmitted terahertz field (see Fig. 2), which is what we measure, is a superposition of the two fields $E_{\mathrm{te}}$ and $E_{\mathrm{to}}$, that is, $E_{\mathrm{t} x}=\left(E_{\mathrm{to}}+E_{\mathrm{te}}\right) / 2$. Hence, the signal intensity is given by

$$
\left|E_{\mathrm{tx}}\right|^{2}=\frac{\left|E_{\mathrm{to}}\right|^{2}+\left|E_{\mathrm{te}}\right|^{2}+2 \operatorname{Re}\left(E_{\mathrm{to}} E_{\mathrm{te}}^{*}\right)}{4}
$$

The last term on the right-hand side represents the interference between the CRA and CRI modes, as observed in one linear-polarization component (say, the $x$ component) of the 

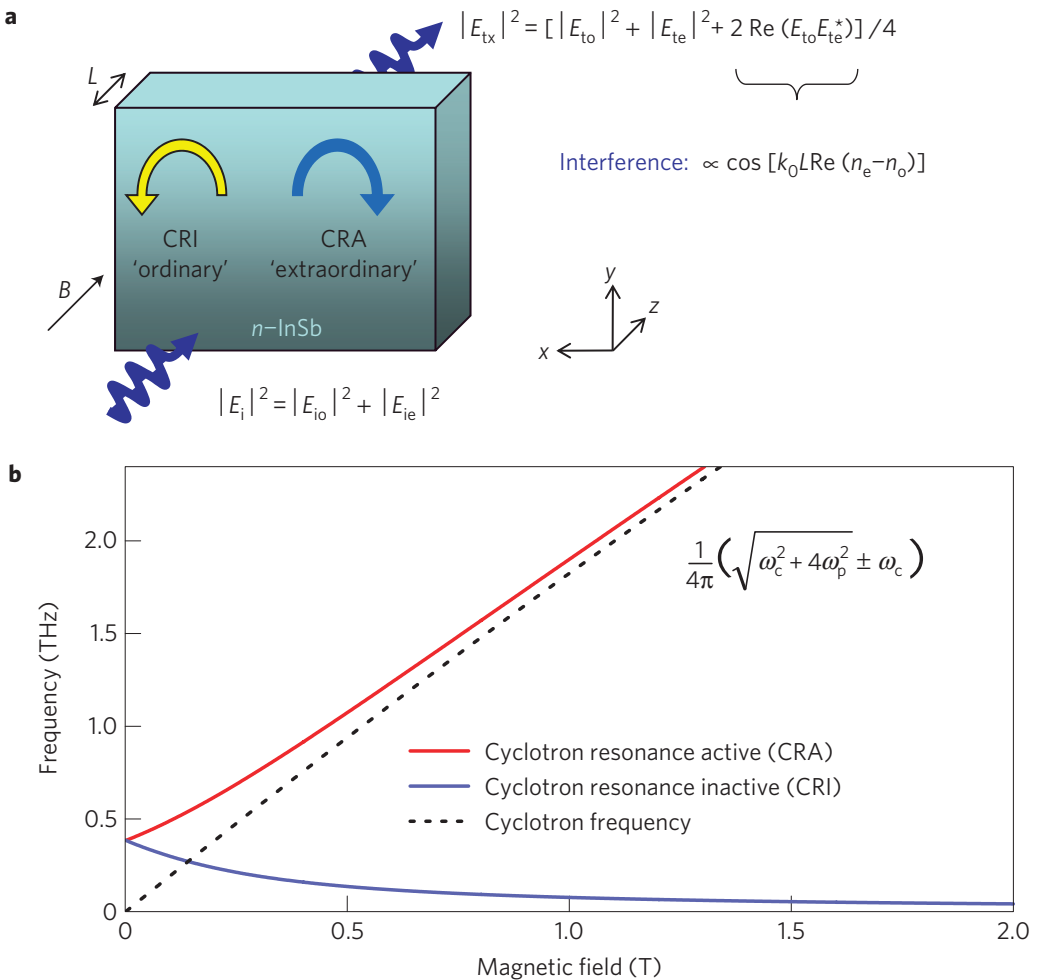

Figure 2 | Interaction of an incident linearly polarized terahertz wave with a plasma in a magnetic field in the Faraday geometry. a, Schematic of the CRA and CRI modes propagating in the magneto-plasma in $n$-InSb in the Faraday geometry. After transmitting through the sample, the $x$ component of the terahertz field has acquired an extra interference term, $\cos \left[k_{0} L \operatorname{Re}\left(n_{\mathrm{e}}-n_{0}\right)\right]$, where $n_{\mathrm{e}}$ and $n_{\mathrm{o}}$ are the extraordinary and ordinary indices of refraction, respectively, $k_{0}$ is the wavenumber in vacuum and $L$ is the sample thickness. $\mathbf{b}$, Frequencies at which the real refractive indices of the CRA and CRI modes are equal to zero (red and blue curves, respectively), given by equation (1), and the electron cyclotron frequency $\omega_{\mathrm{c}} / 2 \pi$ (black dotted curve) as functions of magnetic field. $\omega_{\mathrm{p}} / 2 \pi=0.3 \mathrm{THz}$ is the plasma frequency at zero magnetic field for an electron density of $2.3 \times 10^{14} \mathrm{~cm}^{-3}$.

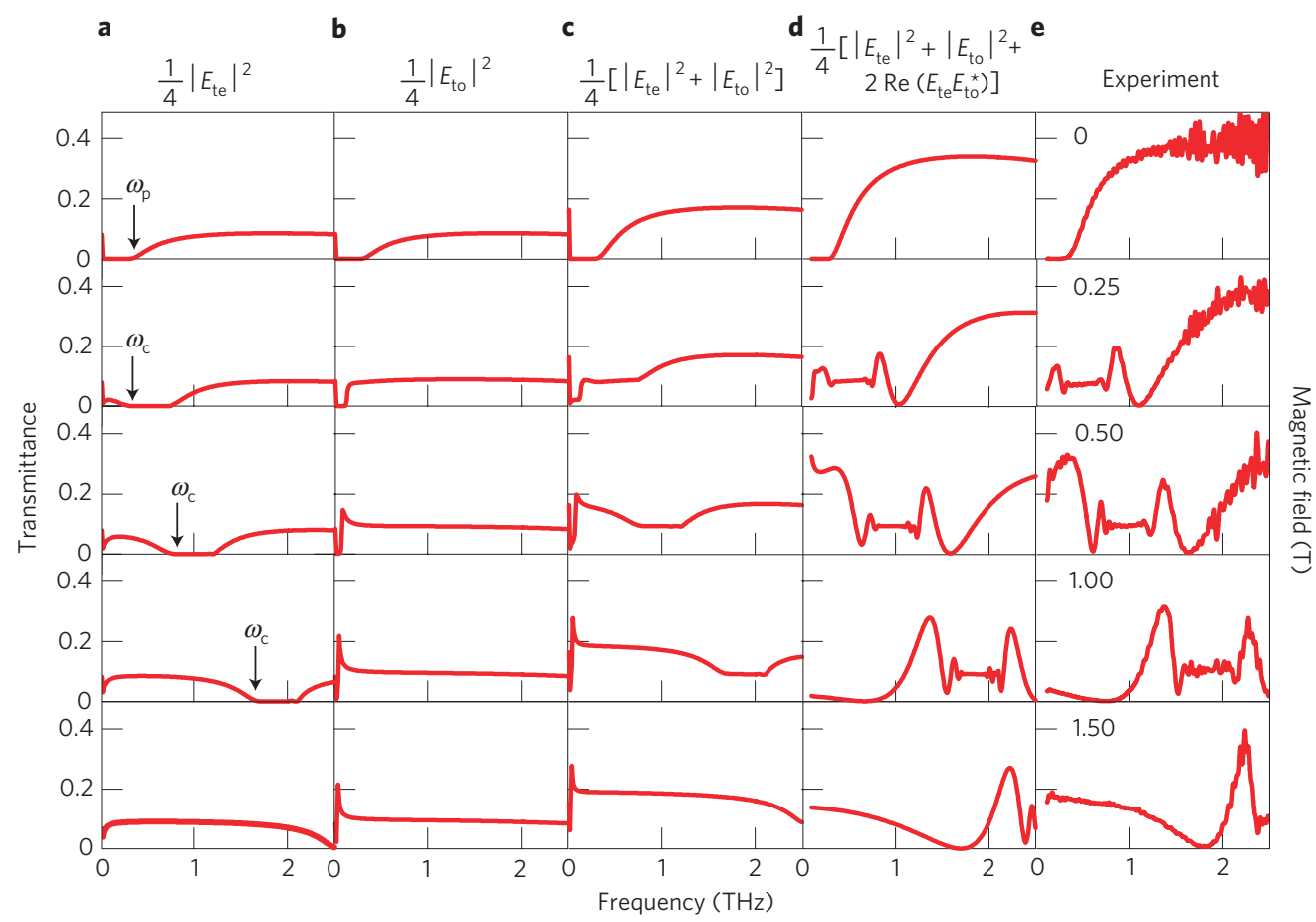

Figure 3 | Experimental and theoretical terahertz transmittance spectra at various magnetic fields. a-d, Calculated terahertz transmittance spectra for $n$-InSb at magnetic fields of $0,0.25,0.5,1$ and $1.5 \mathrm{~T}$ at a temperature of $40 \mathrm{~K}$, including only the CRA wave (a), only the CRI wave (b), CRA and CRI without the interference term (c) and CRA, CRI including the interference term (d). The spectra were calculated using a magneto-plasma model described in the text. e, Experimental terahertz transmittance spectra for $n$-InSb at magnetic fields of $0,0.25,0.5,1$ and $1.5 \mathrm{~T}$ at a temperature of $40 \mathrm{~K}$. All the peaks, dips and plateaux in $\mathbf{e}$, arising from the interference between CRA and CRI modes are reproduced accurately in $\mathbf{d}$. 
a

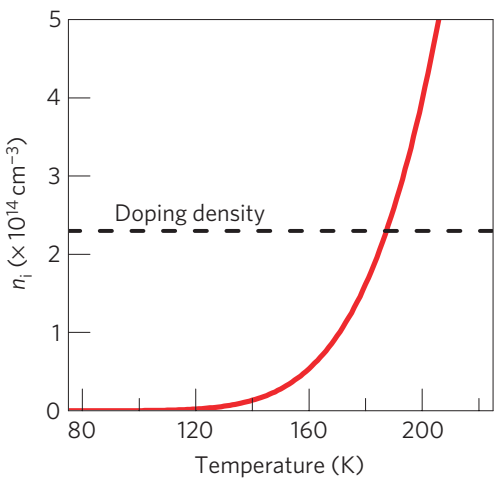

C

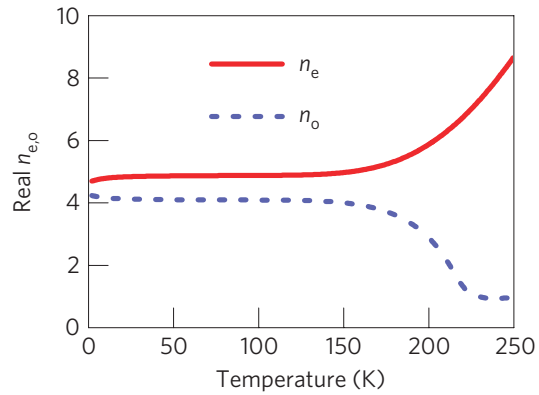

e

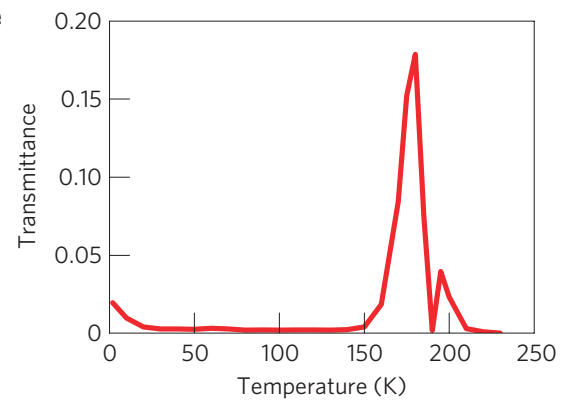

b

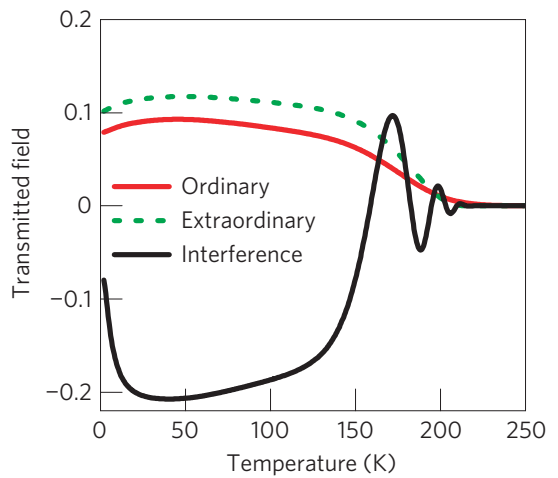

d
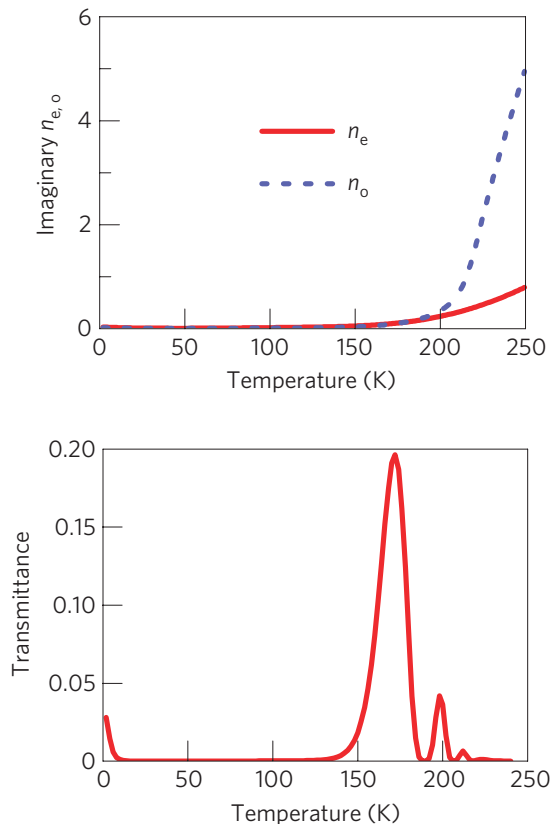

Figure 4 | Appearance of thermally induced terahertz transparency in a magneto-plasma as a result of coherence interference of CRA (extraordinary) and CRI (ordinary) waves. a, Density of intrinsic carriers, $n_{\mathrm{i}}$, versus temperature, excited across the bandgap. The dashed line represents the doping density for the sample $\left(2.3 \times 10^{14} \mathrm{~cm}^{-3}\right)$. $\mathbf{b}$, The three terms of the total transmittance, $\left|E_{\mathrm{tx}}\right|^{2}=\left|E_{\mathrm{te}}\right|^{2}+\left|E_{\mathrm{to}}\right|^{2}+2 \operatorname{Re}\left(E_{\mathrm{te}} E_{\mathrm{to}}^{*}\right)$, as a function of temperature. The last term represents interference between the ordinary and extraordinary waves. $\mathbf{c}, \mathbf{d}$, Real $(\mathbf{c})$ and imaginary $(\mathbf{d})$ parts of the ordinary $\left(n_{0}\right)$ and extraordinary $\left(n_{\mathrm{e}}\right)$ indices of refraction. $\mathbf{e}, \mathbf{f}$, Measured $(\mathbf{e})$ and calculated (f) transmittance of the sample as a function of temperature, corresponding to a horizontal slice of the experimental (Fig. 1b) and theoretical (Fig. 1c) contour maps of transmittance, respectively. All plots in $\mathbf{b}-\mathbf{f}$ are for a frequency of $0.25 \mathrm{THz}$ and a magnetic field of 0.9 T.

transmitted terahertz field through our polarization-selective detection scheme. This term depends on the index difference through $\cos \left[k_{0} L \operatorname{Re}\left(n_{\mathrm{e}}-n_{\mathrm{o}}\right)\right]$, as shown in Fig. 2, where $n_{\mathrm{e}}$ and $n_{\mathrm{o}}$ are the (complex) indices of the CRA and CRI modes, respectively, $k_{0}$ is the wavenumber in vacuum and $L(=0.8 \mathrm{~mm})$ is the sample thickness. When $n_{\mathrm{e}}$ experiences rapid changes owing to electron cyclotron resonance, whereas $n_{\mathrm{o}}$ stays almost constant (see Supplementary Information), the cosine oscillates between -1 and +1 , creating sharp interference fringes. Adding the interference term (that is, $2 \operatorname{Re}\left(E_{\mathrm{te}} E_{\mathrm{to}}^{*}\right)$ ) in our simulation indeed totally modifies the spectra at finite magnetic fields, as shown in Fig. 3d. The agreement between theory (Fig. 3d) and experiment (Fig. 3e) is outstanding. The positions and shapes of all the transmission peaks, plateaux and dips in the spectra are accurately reproduced in great detail, confirming the accuracy of our interpretation and theoretical model and indicating the long coherence times of coupled photonmagneto-plasmon excitations reaching tens of picoseconds.

Next, we consider the striking temperature $(T)$ dependence of the measured terahertz transmittance at a fixed magnetic field, shown in Fig. 1a-c. As the temperature increases, electrical properties of the sample change because of, for example, temperature-dependent scattering processes (see Supplementary Information). However, the dominant process affecting the temperature dependence of the dielectric tensor at elevated temperatures is the thermal excitation of intrinsic carriers across the bandgap given by $n_{\mathrm{i}} \approx 1.1 \times 10^{14} T^{1.5} \exp \left(-E_{\mathrm{g}} / 2 k_{\mathrm{B}} T\right) \mathrm{cm}^{-3}$, which leads to an exponentially growing plasma frequency. Here, $E_{\mathrm{g}} \approx 0.23 \mathrm{eV}$ is the bandgap of InSb at zero temperature. The density of intrinsic carriers $n_{\mathrm{i}}$ eventually exceeds the doping density of $2.3 \times 10^{14} \mathrm{~cm}^{-3}$ at $\sim 180 \mathrm{~K}$, as shown in Fig. $4 \mathrm{a}$. Therefore, one would expect a weakly temperature-dependent transmittance below $\sim 180 \mathrm{~K}$ that would abruptly decrease above this temperature owing to the exponentially growing plasma frequency. The intensities of individually transmitted CRA and CRI modes, $\left|E_{\mathrm{te}}\right|^{2}$ and $\left|E_{\mathrm{to}}\right|^{2}$, respectively, indeed show this expected $T$ dependence, as shown in Fig. $4 \mathrm{~b}$ for a frequency of $0.25 \mathrm{THz}$.

However, as mentioned, the interference term in equation (2), $2 \operatorname{Re}\left(E_{\mathrm{te}} E_{\mathrm{to}}^{*}\right)$, is proportional to $\cos \left[k_{0} L \operatorname{Re}\left(n_{\mathrm{e}}-n_{\mathrm{o}}\right)\right]$. With realistic parameters for our sample and experimental conditions, this interference term is negative and almost exactly cancels the other 
two terms in equation (2) below $160 \mathrm{~K}$, as seen in Fig. 4 b, leading to interference-induced opacity. Here, an incident linearly polarized electric field of unit amplitude $\left(\left|E_{\mathrm{i}}\right|=1\right)$ is assumed, and so the incident amplitudes of the CRA and CRI electric fields are equal to $1 / 2$, that is, $\left|E_{\mathrm{ie}}\right|=\left|E_{\mathrm{io}}\right|=1 / 2$. One can see from Fig. $4 \mathrm{~b}$ that below $160 \mathrm{~K}$ the argument of the cosine function in the interference term, $k_{0} L \operatorname{Re}\left(n_{\mathrm{e}}-n_{\mathrm{o}}\right)$, is nearly constant and is close to $\pi$ for our value of $L$ (see equation (2)). When the temperature increases above $160 \mathrm{~K}$, the difference between the refractive indices of the two normal waves, $n_{\mathrm{e}}-n_{\mathrm{o}}$, starts growing exponentially, causing strong oscillations in the total transmittance owing to the interference term. These oscillations, however, are strongly damped above $200 \mathrm{~K}$ owing to the exponentially growing absorption coefficient for both normal modes, which is proportional to $\omega_{\mathrm{p}}^{2} v / \omega_{\mathrm{c}}^{2}$, where $v$ is the carrier scattering rate. As a result, only one strong peak remains prominent, followed by a few progressively smaller peaks, explaining the existence of the observed transparency bands. This is further illustrated by the excellent agreement in the side-by-side comparison between the observed and calculated temperature dependence of transmittance in Fig. 4e (experiment) and Fig. $4 \mathrm{f}$ (theory) as well as Fig. 1b,e (experiment) and Fig. 1c,f (theory). Thus, we conclude that, counter-intuitively, interference of normal modes causes high opacity of the low-density plasma and creates a transparency window when the plasma density exponentially increases.

These results demonstrate that free-carrier plasmas in lightly doped narrow-gap semiconductors are promising materials systems for terahertz physics, showing huge magnetic anisotropy effects and plasmon excitations in the terahertz range that are highly tunable with external fields, temperature and doping. In particular, we have shown that coherent interference phenomena, which are commonly observed and used in the visible and near-infrared range, can be extended into the terahertz regime. Conventional Fourier-transform infrared spectroscopy should in principle be able to detect these phenomena as long as one measures only one linear-polarization component of the transmitted beam, although multiple-reflection interference fringes might dominate the transmission spectra. Moreover, the observed interference phenomena depend sensitively on plasma properties and carrier interactions, and thus, can be used to study solid-state plasmas over a vast range of external fields and temperatures from the classical limit to the ultra-quantum limit. This experimental finding may open up further new opportunities for using coherent methods to manipulate terahertz waves ${ }^{13,14,19}$ as well as to probe more exotic phenomena in condensed-matter systems that occur owing to many-body interactions and disorder.

\section{Methods}

The time-domain terahertz magneto-spectroscopy system ${ }^{15}$ used in this study consisted of a chirped-pulse amplifier (CPA-2001, Clark-MXR) with a wavelength of $800 \mathrm{~nm}$ and a pulse width of $\sim 200 \mathrm{fs}$ and a pair of $\langle 110\rangle \mathrm{ZnTe}$ crystals to generate and detect coherent radiation from 0.1 to $2.6 \mathrm{THz}$ through surface rectification and electro-optic sampling, respectively. The electro-optic-sampling-based ZnTe detector is polarization-sensitive, and we placed it in such a way that we detect only the horizontal (or $x$ ) component of the transmitted terahertz field, to reveal the magnetic anisotropy-induced interference effects. A shaker, operating at $2 \mathrm{~Hz}$, provided time delays up to $80 \mathrm{ps}$. We averaged over 1,000 scans for each spectrum. We used a magneto-optical cryostat to generate magnetic fields up to $10 \mathrm{~T}$ and varied the sample temperature from 1.6 to $300 \mathrm{~K}$. The sample studied here was a large $\left(\sim 0.8 \times 20 \times 30 \mathrm{~mm}^{3}\right)$ crystal of Te-doped $n$-InSb with an electron density of $2.3 \times 10^{14} \mathrm{~cm}^{-3}$ and a $2 \mathrm{~K}$ mobility of $7.7 \times 10^{4} \mathrm{~cm}^{2} \mathrm{~V}^{-1} \mathrm{~s}^{-1}$. For this particular sample, the Fermi energy at $0 \mathrm{~T}$ was $0.9 \mathrm{meV}$, or $0.21 \mathrm{THz}$, and the (angular) plasma frequency $\omega_{\mathrm{p}}=2 \pi \times 0.28 \mathrm{THz}$. The Faraday geometry was used, where both the propagation direction of a linearly polarized terahertz wave and the magnetic field were perpendicular to the sample surface. We measured the transmitted terahertz waveforms through the sample and an empty hole (as a reference) at each magnetic field at a set of fixed temperatures. Then we Fourier-transformed the time-domain waveforms into the frequency domain and normalized the power spectra to obtain transmittance spectra. To eliminate interference fringes owing to multiple reflections within the sample, only the transmittance spectra of the first pulse that goes directly through the sample are shown in all figures. This windowing procedure effectively reduces the spectral resolution of the measurement to $50 \mathrm{GHz}$, which is more than adequate for the spectral features of interest.

Received 27 June 2009; accepted 10 November 2009; published online 6 December 2009

\section{References}

1. Harris, S. E. Electromagnetically induced transparency. Phys. Today 50, 36-42 (1997).

2. McCall, S. L. \& Hahn, E. L. Self-induced transparency by pulsed coherent light. Phys. Rev. Lett. 18, 908-911 (1967).

3. Zhao, Y., Wu, C., Ham, B., Kim, M. K. \& Award, E. Microwave induced transparency in ruby. Phys. Rev. Lett. 79, 641-644 (1997).

4. Phillips, M. et al. Electromagnetically induced transparency in semiconductors via biexciton coherence. Phys. Rev. Lett. 91, 183602 (2003).

5. Srivastava, A., Srivastava, R., Wang, J. \& Kono, J. Laser-induced above-band-gap transparency in GaAs. Phys. Rev. Lett. 93, 157401 (2004).

6. Zheleznyakov, V. V. Radiation in Astrophysical Plasmas (Kluwer Academic, 1996).

7. Hutchinson, I. H. Principles of Plasma Diagnostics (Cambridge Univ. Press, 1987).

8. Platzman, P. M. \& Wolf, P. A. Waves and Interactions in Solid State Plasmas, Solid State Physics Supplement 13 (Academic, 1973).

9. Litvak, A. G. \& Tokman, M. D. Electromagnetically induced transparency in ensembles of classical oscillators. Phys. Rev. Lett 88, 095003 (2002).

10. Palik, E. D. \& Furdyna, J. K. Infrared and microwave magnetoplasma effects in semiconductors. Rep. Prog. Phys. 33, 1193-1322 (1970).

11. McCombe, B. D. \& Wagner, R. J. in Advances in Electronics and Electron Physics Vol. 37 (ed. Marton, L.) 1-79 (Academic, 1975).

12. Chamberlain, J. M. \& Miles, R. E. (eds) in New Directions in Terahertz Technology (Kluwer Academic, 1997).

13. Tonouchi, M. Cutting-edge terahertz technology. Nature Photon. 1 97-105 (2007).

14. Chen, H.-T. et al. Active terahertz metamaterial devices. Nature 444, 597-600 (2006)

15. Wang, X. et al. Terahertz time-domain magnetospectroscopy of a high-mobility two-dimensional electron gas. Opt. Lett. 32, 1845-1847 (2007).

16. Akhiezer, A. I. Plasma Electrodynamics (Elsevier, 1975).

17. Lifshitz, E. M. \& Pitaevskii, L. P. Physical Kinetics, Course of Theoretical Physics Vol. 10 (Pergamon, 1981).

18. Adachi, S. GaAs and Related Materials (World Scientific, 1994).

19. Avizour, Y. \& Shvets, G. Manipulating electromagnetic waves in magnetized plasmas: Compression, frequency shifting, and release. Phys. Rev. Lett. 100, 065006 (2008).

\section{Acknowledgements}

This work was supported by the National Science Foundation through Award Nos. DMR-0134058, DMR-0325474, ECS-0547019 (CAREER) and OISE-0530220 and the Robert A. Welch Foundation through Grant No. C-1509. We thank A. Srivastava for technical assistance.

\section{Author contributions}

X.W., S.A.C., D.M.M. and J.K. carried out the terahertz measurements. A.A.B. developed the theoretical model and carried out theoretical simulations. All authors analysed the experimental data and contributed to the preparation of the manuscript.

\section{Additional information}

The authors declare no competing financial interests. Supplementary information accompanies this paper on www.nature.com/naturephysics. Reprints and permissions information is available online at http://npg.nature.com/reprintsandpermissions. Correspondence and requests for materials should be addressed to J.K. 